\title{
NEPHROTIC SYNDROME AS PARANEOPLASTIC SYNDROME IN A PATIENT WITH THYMIC MASS
}

\author{
Satyabrata Guru1, Rashmi Ranjan Mohanty², Debasish Das ${ }^{3}$
}

${ }^{1}$ Senior Resident, Department of General Medicine, AIIMS, Bhubaneswar.

${ }_{2}^{2}$ Associate Professor, Department of General Medicine, AIIMS, Bhubaneswar.

${ }^{3}$ Assistant Professor, Department of Cardiology, AIIMS, Bhubaneswar.

\begin{abstract}
\section{BACKGROUND}

Paraneoplastic glomerulonephritis is a rare complication of malignancy that is frequently mistaken for idiopathic glomerulonephritis. Failure to recognise paraneoplastic glomerulonephritis can subject patients to ineffective and potentially harmful therapy. ${ }^{1}$ We report a case who presented with SOB and generalised swelling. On investigations found to have Nephrotic syndrome and hilar mass, on HRCT Thorax Thymic mass was found. Final diagnosis of 'Nephrotic Syndrome as Paraneoplastic syndrome in a patient with Thymic mass' was made. The pathophysiology, approach to the diagnosis and mode of treatment are also discussed. Despite the rarity of this association, this clinical observation underlines that a thymoma should be searched in the presence of a glomerulopathy. The glomerulopathy can be improved by the treatment of the thymoma.
\end{abstract}

\section{KEYWORDS}

Paraneoplastic, Nephrotic, Thymoma.

HOW TO CITE THIS ARTICLE: Guru S, Mohanty RR, Das D. Nephrotic syndrome as paraneoplastic syndrome in a patient with thymic mass. J. Evolution Med. Dent. Sci. 2017;6(32):2670-2671, DOI: 10.14260/Jemds/2017/575

\section{BACKGROUND}

Paraneoplastic glomerulonephritides are glomerular lesions that are not directly related to tumour burden, invasion or metastasis, but rather are induced by products from tumour cells. ${ }^{2}$ The first series of paraneoplastic glomerulonephritis was published over 40 years ago by Lee et al. ${ }^{3}$ In general, a diagnosis of paraneoplastic glomerulonephritis should be considered if the glomerulonephritis occurs in the presence of malignancy, remits after ablation of malignancy and recurs in association with the recurrence of malignancy. 4

\section{CASE REPORT}

A 45-year-old male was admitted with c/o progressive generalised swelling- 5 days and SOB- 2 days. It was not associated with decreased urination, haematuria or chest pain. 0/E- Patient was dyspnoeic, oedema and abdominal distention present, vitals-stable. On routine investigationsurine shows 4+ albuminuria, hyperlipidaemia, hypoalbuminaemia, 24 hrs. urine protein- $12.7 \mathrm{gm}$, hypothyroidism, $\mathrm{x}$-ray chest shows- rt. hilar mass with $\mathrm{b} / \mathrm{l}$ pleural effusion. Patient treated in line of Nephrotic syndrome with Inj. Lasix, Omnacortil and Thyroxine (50 mcg). Patient's dyspnoea improved by only $10 \%-15 \%$. Subsequently, HRCT thorax was done which shows Thymic mass. Final diagnosis of 'Nephrotic Syndrome as a Paraneoplastic syndrome in a patient with Thymic mass' was made and planned for Thymectomy with excisional biopsy.

Financial or Other, Competing Interest: None.

Submission 27-03-2017, Peer Review 13-04-2017,

Acceptance 15-04-2017, Published 20-04-2017.

Corresponding Author:

Satyabrata Guru,

Department of General Medicine,

AIIMS, Bhubaneswar,

Odisha.

E-mail: satyabrataguru@yahoo.com

DOI: $10.14260 /$ jemds $/ 2017 / 575$

\section{(c) $(i) \$$}

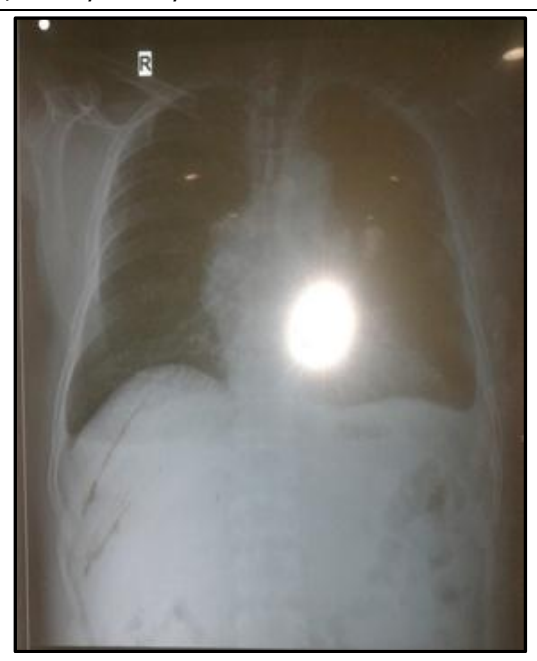

Figure 1. Rt. Hilar Mass with B/L Pleural Effusion

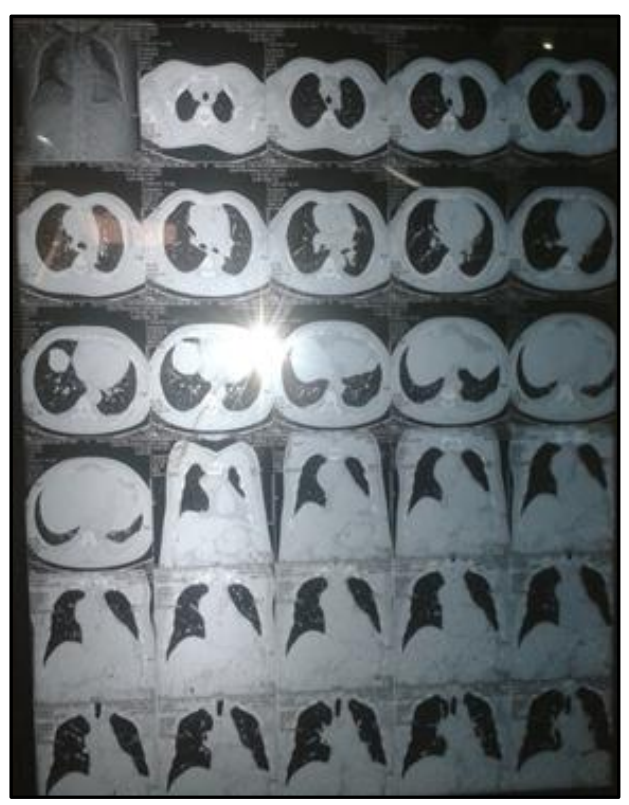

Figure 2. Thymic Mass 


\section{DISCUSSION}

Thymoma is a rare malignancy that is unique because of its association with a variety of autoimmune diseases including myasthenia gravis, systemic lupus erythematosus, pure red cell aplasia and pemphigus vulgaris. This association implies that severe T-cell dysregulation occurs in patients with thymoma- a feature that may be implicated in the pathogenesis of thymoma-associated paraneoplastic glomerulonephritis. The prevalence of paraneoplastic glomerular diseases in patients with thymoma is about $2 \%$. Histologically, thymoma can be divided into epithelial predominant or lymphocyte predominant tumours. Membranous nephropathy tends to be associated with epithelial predominant thymoma, is always diagnosed with either newly diagnosed or recurrent thymoma and typically resolves after tumour ablation. MCD tends to be associated with lymphocyte-predominant thymoma, is frequently diagnosed after tumour removal and responds relatively well to steroid therapy (with a combined complete and partial remission rate of $60 \%$ ). Thymoma-associated membranous nephropathy seems to have a similar pathogenetic mechanism to that of solid tumour-associated membranous nephropathy, whereas thymoma-associated MCD could be associated with persistent T-cell dysfunction after thymoma removal. 5

\section{CONCLUSION}

Paraneoplastic glomerulonephritis, a rare secondary cause of glomerulonephritis and a complication of cancer, remains a challenge to both nephrologists and oncologists. Altered immune responses seem to play a role in the pathogenesis of paraneoplastic glomerulonephritis. Recognition of paraneoplastic glomerulonephritis and subsequent detection of an undiagnosed malignancy could be life-saving. The treatment of paraneoplastic glomerulonephritis is targeted at the cause of the neoplasm and requires a multidisciplinary approach to monitor both the cancer and the glomerular lesions.

\section{REFERENCES}

[1] Lien YH, Li-Wen L. Pathogenesis, diagnosis and management of paraneoplastic glomerulonephritis. Nature reviews Nephrology 2011;7(2):85-95.

[2] Ronco PM. Paraneoplastic glomerulopathies: new insights into an old entity. Kidney Int 1999;56(1):35577.

[3] Lee JC, Yamauchi H, Hopper J. The association of cancer and the nephrotic syndrome. Ann Intern Med 1966;64(1):41-51.

[4] Bacchetta J, Juillard L, Cochat P, et al. Paraneoplastic glomerular diseases and malignancies. Crit Rev Oncol Hematol 2009;70(1):39-58.

[5] Karras A, de Montpreville V, Fakhouri F, et al. Renal and thymic pathology in thymoma-associated nephropathy: report of 21 cases and review of the literature. Nephrol Dial Transplant 2005;20(6): 1075-82. 